MKG-Chirurg 2012 $\cdot 5: 265-265$

DOI 10.1007/s12285-012-0299-6

Online publiziert: 1. November 2012

c) Springer-Verlag Berlin Heidelberg 2012

H.-F. Zeilhofer

Klinik für Mund-, Kiefer- und Gesichtschirurgie, Universitätsspital Basel

\title{
Kraniofaziale Chirurgie
}

\section{Interdisziplinarität und innovative Technologien}

den; das betrifft den einzelnen Chirurgen ebenso wie unsere Fachgesellschaft.

die kraniofaziale Chirurgie ist zu einem integralen Bestandteil unseres Fachgebiets geworden. Das spiegelt sich in Lehre, Forschung und Krankenversorgung wider und ist in unserer Weiterbildungsordnung gesetzlich geregelt. Die kraniofaziale Chirurgie wird aber auch von Spezialisten anderer Fachgebiete, wie der Kinderchirurgie, der Neurochirurgie oder der HNOHeilkunde, durchgeführt.

Die Fortschritte in der Anästhesiologie und Intensivmedizin sowie der Pioniergeist von Paul Tessier in den 1960er Jahren hatten dazu geführt, dass sich diese spezielle Disziplin entwickeln und etablieren konnte. Im Laufe der prosperierenden Entwicklung der kraniofazialen Chirurgie ist der mutige Pionier dann vom interdisziplinären Zentrum abgelöst worden, in das sich die Mund-, Kiefer- und Gesichtschirurgie mit den ihr immanenten Stärken einbringt.

Ein modernes interdisziplinäres Zentrum sollte in enger Beziehung zu universitären Forschungseinrichtungen stehen, denn die Patienten mit kraniofazialen Fehlbildungen haben von den innovativen Technologien wie Bildgebung, 3-DPlanung, modellbasierter Chirurgie, Navigation oder der Distraktionsosteogenese sehr profitiert.

Die für eine moderne MKG-Chirurgie notwendige Interdisziplinarität muss zwischen den Polen Medizin und Zahnmedizin in einem permanenten Prozess immer wieder neu definiert und gelebt wer-

\section{1) Die notwendige Interdisziplinarität muss immer wieder neu definiert und gelebt werden}

Auch die MKG-Chirurgie hat ihr Gesicht in den letzten Jahrzehnten verändert. Stand früher das Gemeinsame mit der Zahnheilkunde im Vordergrund, so wird heute die Verbindung mit den medizinisch-chirurgischen Disziplinen immer wichtiger. Sorgte früher vor allem die dentale Werkstoffkunde für Innovationen, so bestimmen heute Informatik, Bioengineering und neue Materialien die kreative Zukunft der MKG-Chirurgie.

Es ist kein Zufall, sondern Programm, dass den State-of-the-Art-Artikel zur kraniofazialen Chirurgie ein Neurochirurg, H. Haberl, und ein MKG-Chirurg, N. Adolphs, gemeinsam verfasst haben.

Wie für seltene Erkrankungen mit kraniofazialer Manifestation besondere Lösungswege in enger Kooperation mit naturwissenschaftlich-technischen Disziplinen und der Industrie entwickelt werden, demonstriert uns $P$. Jürgens. Die neuen Methoden wurden standardisiert und erweisen sich als nützlich in der täglichen Routine.

Der MKG-Chirurg C. Blecher beschreibt für alle verständlich die Prinzipien der Therapie von lagebedingten Schädeldeformitäten. Dabei kommen meh- rere innovative Technologien zum Einsatz: 3-D-Oberflächenscanning, virtuelles Planen und Rapid Manufacturing. Diese Technologien benötigen aber auch das verbindende Handling des Chirurgen. Der fertige Hightechhelm wird vom erfahrenen Chirurgen mit Mikromotor und rotierenden Instrumenten bearbeitet.

Ich hoffe, Sie, die Leser dieser Ausgabe, sehen die Bedeutung von Interdisziplinarität und Orientierung an innovativen Technologien für die MKG-Chirurgie in einem neuen Licht.

Ihr

Gans. Forth bille Yer

Hans-Florian Zeilhofer

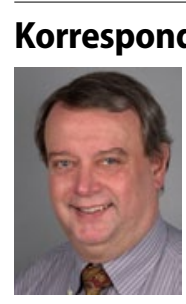

Prof. Dr. Dr. Dr. h. c.

H.-F. Zeilhofer Klinik für Mund-, Kieferund Gesichtschirurgie, Universitätsspital Basel Spitalstr. 21, 4031 Basel Schweiz hfzeilhofer@uhbs.ch 